\section{Percepcão de mulheres com hiperténsão sobre a prática de ioga na saúde e na qualidade de vida}

\section{Perception of women with hypertension on yoga practice in health and quality of life}

Julio Mizuno

Fernanda Rossi ${ }^{2}$

Silvia Deutsch'

Henrique Luiz Monteiro

\section{RESUMO}

O objetivo deste estudo foi explorar as percepções de mulheres adultas e idosas com hipertensão arterial participantes de um programa de exercícios de Ioga. Para coleta de dados foi utilizada a técnica de grupo focal com questionário semiestruturado, que permitiu extrair opiniões e sentimentos, compartilhando diferentes visões de um mesmo fenômeno. Os relatos foram transcritos, analisados pelo método de análise de conteúdo e divididos em quatro categorias, cujos resultados foram: a) Dimensão da prática: a participação no programa melhorou os hábitos alimentares e a disposição para realizar atividades físicas, que estão relacionados com o autocuidado e a conscientização; b) Dimensão física: os relatos apontam para a melhora em realizar atividades cotidianas, indicando melhoras das capacidades físicas, proporcionados pelos exercícios de Ioga; c) Dimensão saúde-doença: observamos a redução das queixas de dores musculares, articulares, do consumo de medicação e de sinais e sintomas; e, d) Dimensão psicossocial: melhoras no estado de humor, autoconhecimento, autoestima e reflexões do âmbito social. O programa de Ioga pode proporcionar diversos benefícios às participantes, apontando para a existência de uma perspectiva em saúde, observável a partir das percepções expressas no grupo focal, que podem ser descritas em seus prontuários clínicos.

\section{PALAVRAS-CHAVE}

Hipertensão; Ioga; Qualidade de vida.

\begin{abstract}
The objective of this study was to explore the perceptions of adults and elderly women with bypertension participants of a yoga exercise program. For data collection was used the focus group technique with semi-structured questionnaire, which allowed to extract opinions and feelings, sharing different views of the same phenomenon. The reports were transcribed and analyzed by content analysis method and divided into four categories, the results were: a) Practical dimension: participation in the program improved eating habits and willingness to perform physical activities, which are related to self-care and awareness; $b$ ) Physical dimension: the reports point to the improvement in performing daily activities, indicating improvement of physical, provided by yoga exercises; c) Health and disease dimension: we saw a reduction of complaints of muscle pain, joint, medication consumption and signs and symptoms; and, d) Psychosocial Dimension: improvements in mood, self-knowledge, self-esteem and reflections of the social sphere. The yoga program can provide many benefits to the participants, pointing to the existence of a bealth perspective, observable from perceptions expressed in the focus group, that can be described in the medical records.
\end{abstract}

\section{KEYWORDS}

Hypertension, Yoga, Quality of Life.
Rev Bras Ativ Fís Saúde p. 376-385 DOI

http://dx.doi.org/10.12820/rbafs.v.20n4p376

1 Unesp, Depto de Educação Física, Rio Claro, $S P$, Brasil.

2 Unesp, Depto de Educação, Bauru, SP, Brasil. 


\section{INTRODUÇÃO}

As práticas corporais complementares de origem oriental, como a Ioga, têm se inserido no campo da saúde tanto no contexto clínico quanto epidemiológico, atuando nos cuidados com o corpo, como terapia adjuvante no tratamento convencional das mais variadas doenças ${ }^{1}$.

A Ioga é uma prática milenar originária da Índia, introduzida no ocidente há mais de um século que engloba conceitos filosóficos, posturas físicas, exercícios de respiração, exercícios de concentração e práticas de meditação, com o objetivo de conduzir o ser humano ao autoconhecimento, liberdade de condicionamentos, felicidade plena e transcendência ${ }^{2}$. É popularmente conhecida por reduzir o estresse, melhorar o humor, o condicionamento físico, promover bem estar de forma geral e a qualidade de vida de seus adeptos ${ }^{3}$.

Pesquisas clínicas com as técnicas de Ioga aplicadas de forma isolada ou combinada, em diferentes condições de saúde têm demonstrado efetividade no tratamento da hipertensão arterial $^{4}$, diabetes ${ }^{5}$, depressão ${ }^{6}$, ansiedade ${ }^{7}$ e insônia ${ }^{8}$. No entanto, considerando que a Ioga é uma prática corporal, não se pode limitar seus benefícios apenas aos efeitos fisiológicos e psíquicos medidos quantitativamente, sem se ater ao que pensa e pratica o indivíduo ou grupo, abordando a questão da perspectiva integral do ser humano. Dessa forma, pesquisas de natureza qualitativa podem contribuir para a compreensão dos benefícios advindos da Ioga sobre a perspectiva dos praticantes.

A vivência de Ioga ofertada como atividade de extensão universitária na Universidade Estadual Paulista, campus de Bauru, com mulheres portadoras de hipertensão arterial, usuárias do serviço público de saúde municipal, permitiu constatar que além dos resultados favoráveis demonstrados através de dados quantitativos ${ }^{4}$, as participantes relatavam acontecimentos e situações de vida diária que atestavam benefícios nos aspectos gerais relacionados à qualidade de vida. Estas mudanças faziam referência ao estilo de vida com adoção de hábitos saudáveis e maior atenção com o autocuidado, o que despertou o interesse dos pesquisadores responsáveis para a necessidade de estudos que contemplassem o conteúdo desses relatos.

Em face desta constatação, o objetivo do presente estudo foi explorar as percepções de mulheres adultas e idosas com hipertensão arterial participantes de um programa de exercícios de Ioga.

\section{MÉTODOS}

Para investigar os benefícios da Ioga, na perspectiva das praticantes, a pesquisa pautou-se nos pressupostos da abordagem quantitativa, com dados clínicos, seguida de uma abordagem qualitativa, que segundo Ginzburg ${ }^{9}$, um método investigativo centrado nas raízes do conhecimento das Ciências Humanas e Sociais é indireto, indiciário, conjetural; consiste na análise de minúcias, detalhes que são os verdadeiros reveladores dos significados para a pesquisa. Esta perspectiva coloca o pesquisador como o principal instrumento de coleta, estabelecendo o contato prolongado com os participantes investigados, sendo essencial estabelecer uma visão holística dos fenômenos. Fundamentada nesse pressuposto, a pesquisa se estabeleceu com um grupo de mulheres adultas e idosas, participantes regulares de um Programa de Ioga. 
O Programa de Extensão loga e Hipertensão e as participantes do estudo

Participaram da pesquisa doze mulheres com idade entre 46 e 74 anos, usuárias de duas unidades básicas de saúde da cidade de Bauru sob tratamento regular da hipertensão arterial há pelo menos um ano, sem restrição médica para a prática de atividade física e que frequentaram pelo menos $75 \%$ das aulas do programa "Ioga e Hipertensão", oferecido pelo Laboratório de Avaliação e Prescrição de Exercícios do Departamento de Educação Física da Unesp , por um período de quatro meses. Antes de iniciarem as atividades, as participantes responderam uma anamnese com dados pessoais, histórico clínico e hábitos de vida e passaram por avaliação antropométrica e clínica.

As mulheres tinham à disposição transporte gratuito entre as unidades de saúde e o campus. Antes do início da aula, os monitores aferiam a pressão arterial e a frequência cardíaca para controle e medida de segurança.

As aulas fundamentaram-se nos oito passos da Ashtanga Yoga de Patãnjali, que inclui princípios filosóficos, posturas de Ioga, aprendizagem dos exercícios de respiração, concentração e meditação associadas a técnicas de relaxamento em três sessões semanais, com duração de 90 minutos. As sessões foram elaboradas pelo mediador do programa e pesquisador deste estudo, formado em Licenciatura Plena em Educação Física e professor de Ioga há 12 anos. Além das aulas, mensalmente realizava-se uma palestra com temas variados relacionados à saúde, seguido de café da manhã comunitário, no qual as participantes levavam alguma contribuição (bolos, pães, salgados, etc.).

O projeto de pesquisa foi aprovado pelo Comitê de Ética em Pesquisa da Faculdade de Ciências da Unesp de Bauru (Parecer: 254.378) e seguiu a resolução n ${ }^{\circ}$ 466/12 do Conselho Nacional de Saúde. A participação das mulheres foi voluntária e poderia encerrar-se a qualquer momento, se assim desejassem. Tanto essa informação quanto os objetivos, os procedimentos de coleta de dados, a garantia do anonimato (com a atribuição de códigos ou nomes fictícios quando da divulgação dos resultados) e a autorização para a publicação dos dados fornecidos apenas para fins científicos constaram do Termo de Consentimento Livre e Esclarecido assinado pelas participantes.

\section{Técnicas de Pesquisa}

Para identificar os benefícios da prática de Ioga na percepção das praticantes, utilizou-se a técnica de grupo focal para a coleta de dados. A técnica permite "emergir uma multiplicidade de pontos de vista e processos emocionais, pelo próprio contexto de interação criado, permitindo a captação de significados que, com outros meios, poderiam ser difíceis de manifestar" ${ }^{10}$.

A sessão de grupo focal foi realizada após quatro meses de Programa de Ioga. A duração da entrevista foi de 90 minutos e os relatos foram gravados em áudio. Atentou-se para que os aparelhos fossem minimamente invasivos de modo que as participantes não se sentissem inibidas ou constrangidas. Posteriormente os dados foram transcritos na íntegra.

O grupo focal permite promover insights, como ressalta Morgan ${ }^{11}$, na medida em que os participantes, ao expressarem suas opiniões e sentimentos, podem se dar conta de crenças e atitudes presentes tanto em seus comportamentos quanto dos outros, compartilhando diferentes visões de um mesmo fenômeno.

As informações, argumentos, crenças e discussões durante uma sessão de grupo focal podem revelar pensamentos individuais, bem como resultar na com- 
preensão coletiva e análise crítica das questões colocadas em debate. Ainda, de acordo com Patton ${ }^{12}$, esta técnica revela-se eficiente, dentre outras razões, por apresentar-se mais fielmente às percepções dos participantes que tendem a refletir sobre si mesmos na dinâmica em grupo, possibilitando verificar visões compartilhadas e consistentes.

Baseado nesses pressupostos buscou-se, desencadear a reflexão e a compreensão das participantes em relação à prática da Ioga por intermédio de suas próprias interpretações.

Os temas de orientação para estimular a discussão na sessão de grupo focal foram organizados previamente, da seguinte forma: i) abordagem para participação no projeto; ii) conhecimento sobre Ioga; iii) opinião sobre os exercícios de Ioga realizados; iv) ambiente da universidade; v) atividades diárias; vi) interação entre professores, monitores e participantes; vii) mudanças observadas no dia a dia após o programa; viii) observação em relação à saúde após o programa; e, ix) utilização dos exercícios no dia a dia. Os relatos não seguiram esta sequência, foram surgindo durante a discussão juntamente com outros temas emergentes não previstos. Quando necessário os entrevistadores estimularam a fala das participantes baseados nos temas de orientação, sendo que ao final da entrevista verificou-se se todos os tópicos foram discutidos pelo grupo.

\section{Análise dos dados}

O material empírico obtido por meio de grupo focal foi organizado e categorizado procurando identificar tendências e padrões relevantes.

A análise dos dados se embasou nos pressupostos da análise de conteúdo que Bardin ${ }^{13}$ (p. 38) define como "um conjunto de técnicas de análise das comunicações, que utiliza procedimentos sistemáticos e objetivos de descrição do conteúdo das mensagens", sendo pertinente para o estudo das motivações, atitudes, valores, crenças e tendências do grupo pesquisado. Especificamente em relação à pesquisa com grupos focais, Morgan ${ }^{11}$ ressalta que os dados obtidos podem ser analisados e interpretados de diferentes formas, de acordo com o objetivo da pesquisa, sendo a análise de conteúdo um dos métodos mais utilizados.

A partir da análise do material empírico obtido foi realizada classificação, codificação e categorização ${ }^{14}$ dos relatos nas seguintes categorias: a) Dimensão da prática; b) Dimensão física; c) Dimensão saúde-doença; e, d) Dimensão psicossocial. Cada relato foi classificado apenas na dimensão que mais se aproximava, independente dos temas de orientação do qual foi extraído.

\section{RESULTADOS E DISCUSSÃO}

\section{Características das participantes}

As participantes apresentavam idades entre 46 e 74 anos e realizavam tratamento regular para hipertensão arterial e outras doenças crônicas (diabetes [17\%], dislipidemias [17\%] e hipotireoidismo [50\%]), razão pelo qual 83\% consumiam pelo menos dois medicamentos de uso contínuo. O índice de massa corporal médio foi de $29,5 \pm 5 \mathrm{Kg} / \mathrm{m}^{2}$ (sobrepeso); $75 \%$ eram da classe econômica $\mathrm{C}$ e $67 \%$ informaram possuir ensino fundamental completo.

A pressão arterial sistólica no início do programa era de $134 \pm 10 \mathrm{mmHg}$ e reduziu para $122 \pm 7 \mathrm{mmHg}(\mathrm{p}<0,001)$, enquanto a pressão arterial diastólica 
$(83 \pm 12$ versus $80 \pm 11 \mathrm{mmHg} ; \mathrm{p}>0,05)$ e a frequência cardíaca $(77 \pm 7$ versus $80 \pm 10 ; p>0,05)$ permaneceram similares entre os dois momentos, corroborando com outros estudos na literatura ${ }^{4,5}$.

\section{Dimensão da prática}

Nesta dimensão foi abordado o conhecimento prévio das participantes sobre a Ioga, as percepções durante a participação nas atividades dentro da universidade e as mudanças de comportamento/hábito advindos da prática regular de Ioga.

A Ioga tem se popularizado nos últimos anos com a divulgação da mídia impressa e visual e nos clubes e academias que a oferecem. Desta forma algumas participantes já a conheciam: "Eu fiz ioga uma vez lá no SESC", "eu já tinha feito com o professor mesmo"; enquanto que outras apenas tinham "ouvido falar" ou "já tinha visto, mas não sabia como era"; algumas tinham um conceito equivocado sobre a Ioga: "eu pensava que a ioga era só ficar assim", referindo-se à posição sentada de meditação ou confundindo com o Tai-chi, uma prática corporal chinesa. A maioria nunca tinha participado de uma aula e apresentava conceitos incompletos sobre o que se tratava a Ioga, pois acreditavam que os exercícios se restringiam à meditação e aos exercícios de respiração. Tais concepções podem ser compreendidas ao analisar-se o propósito original da Ioga que esta ligada ao aspecto espiritual da existência humana, entretanto, no ocidente, para os profissionais que atuam com Ioga a prática tem se incorporado à cultura como um tipo de ginástica para a saúde e a redução do estresse ${ }^{2}$, no entanto, como pode observar-se, a percepção das mulheres dessa faixa etária ainda é bem diferente daquela que tem os especialistas em Ioga.

A rotina do programa incluía controle diário da pressão arterial; orientação do uso correto das medicações prescritas (doses e horários); palestras mensais com temas variados (nutrição, psicologia, primeiros socorros, etc) e avaliações semestrais conduzidos pelos monitores do projeto (alunos do curso de Educação Física), fazendo com que as participantes se sentissem acolhidas e valorizadas: "Os meninos (monitores) todos dedicados, são muito atenciosos, educados". De fato, o elemento social é fundamental para a melhoria da qualidade de vida no âmbito das práticas corporais, segundo Castellano et al. ${ }^{15}$, programas de atividades físicas podem proporcionar o acolhimento social, a convivência com pessoas de outras gerações e a percepção de suporte, muitas vezes não encontrada no ambiente doméstico e no sistema público de saúde.

Os relatos indicam que a participação no programa incentivou o aumento das atividades físicas de forma geral: "eu faço o exercício em casa também", "a ioga eu faço aqui e em casa e também faço caminhada"; e também mudanças positivas nos hábitos alimentares: "emagreci quatro quilos, associado com a dieta", "tirei carne, fritura, açúcar, etc.", "estou bebendo mais água", demonstrando que a participação no programa promoveu mudanças de hábitos com adoção de comportamentos favoráveis, relacionados ao autocuidado e a conscientização. As características das aulas de Ioga propicia o autoconhecimento que por sua vez estimula o autocuidado e a adoção de hábitos de vida mais saudáveis ${ }^{3}$.

\section{Dimensão Física}

Nesta dimensão estão inseridos os relatos associados aos benefícios físicos proporcionados pelos exercícios, tais como a melhora de capacidades físicas e do desempenho em atividades de vida diária e laborais. 
A melhora das capacidades físicas foram as mais citadas no grupo focal, como aumento da flexibilidade, da força, do tônus muscular e do equilíbrio. Estas capacidades podem ser estimuladas e desenvolvidas com os exercícios de Ioga, que são compostos de alongamento, posturas em contração isométrica e de estabilidade postural ${ }^{16}$. Após quatro meses as participantes relataram: "nеm colocar a mão no chão eu conseguia, agora está voltando", "depois que faço assim sinto alongado, muito alongamento"; "agilidade de abaixar", "parece que ativa tudo, então você fica com músculo mais forte", "ai está tudo endurecendo de novo (musculatura)" e também no equilíbrio. Cowen e Adams ${ }^{17}$ também observaram melhora significativa da flexibilidade e da força após seis semanas de práticas regulares de Ioga em uma amostra de 26 adultos.

Apesar da maioria dos exercícios de Ioga apresentar baixa intensidade física $^{18}$ a condição inicial das participantes caracterizada por baixa atividade física, possibilitou melhoras perceptíveis no condicionamento físico, refletindo no aumento da disposição para realização das atividades de vida diária conforme enfatizam nos relatos: "Eu consigo limpar a minha casa sozinha, e é grande a minha casa, são quatro quartos, dois banbeiros, duas salas..."; "Fazia um pouquinho já cansava, agora não. Faço um pouco, no outro dia mais um pouco. Mas eu não tenho mais aquela canseira...”. Apesar da maioria das participantes serem aposentadas ou do lar, duas delas estavam inseridas no mercado de trabalho e relataram benefícios advindos da prática de Ioga: "eu voltei a trabalhar depois que eu comecei no Ioga, eu voltei e sai da licença médica, eu voltei a trabalhar, tudo por causa do Ioga..."; e outra: "Eu trabalho em pé, eu tomava remédio todo dia pra poder aguentar [...] mas depois que eu comecei é raro, bem raro e eu tinha que tomar [remédio] todo dia se não, não conseguia trabalhar". A melhora da autonomia funcional foi observada também em idosos praticantes de Ioga avaliados pelo protocolo de LADEG ${ }^{19}$.

Sobre a dimensão física, muitas vezes as pesquisas não expressam diferenças estatísticas nos valores de média e desvio padrão de testes e avaliações entre momentos. Neste caso, a pesquisa qualitativa identifica mudanças pontuais que não são perceptíveis pelos métodos cartesianos de pesquisa científica, mostrando a importância e a necessidade de utilização da informação qualitativa na pesquisa e na abordagem clínica dos pacientes de tal forma que esses possam complementar os dados quantitativos oriundos dos métodos tradicionais de abordagem do processo saúde-doença e que sejam registradas no prontuário clínico do paciente pelo corpo médico, de enfermagem ou pelo profissional de educação física.

\section{Dimensão Saúde-Doença}

Nesta dimensão estão agrupadas as percepções relacionadas à melhora da condição de saúde, como a redução de sinais e sintomas associados ou não a patologias e redução do consumo de medicação.

A redução no quadro de dor foi uma das mais observadas entre os relatos: "Meu joelho melhorou bem, dói um pouco ainda", "Eu tinha muita dor nas costas também, agora é raro, mas antes era constante, eu tinha muita dor e dificuldade", e "até para subir uma escada, um degrau eu tinha dificuldade por causa do joelho". Os exercícios de Ioga que as participantes realizaram constituem um conjunto de procedimentos que podem auxiliar no alongamento muscular e na circulação sanguínea, contribuindo assim para reduzir o quadro de dores lombares ${ }^{20-21}$. Os 
quatro meses de aulas proporcionaram efeitos positivos na percepção da dor, o que contribuiu para que elas adotassem os exercícios como rotina diária. Além das dores musculares, houve redução também na frequência de cefaleias: " $E u$ tinha muita dor de cabeça", que pode ser efeito de um estado geral de relaxamento, com redução da tensão e consequentemente das dores de cabeça de origem tensional. Uma revisão de literatura realizada por Bussing et al. ${ }^{22}$ concluiu que a Ioga pode ser usada para inúmeras condições associadas a algias.

Outra observação importante foi a redução da pressão arterial, já que as participantes eram hipertensas e mesmo realizando o tratamento medicamentoso prescrito, ainda assim, algumas não conseguiam controlar a pressão arterial: "Eu ficava de madrugada comendo alho para abaixar a pressão, tomava remédio" ou "a minha pressão era 17, 16 [...] agora a pressão está boa, 11, 12, 13". De fato, esse efeito hipotensor dos exercícios foi observado por Mizuno e Monteiro ${ }^{4}$ que utilizaram a Ioga como proposta complementar de tratamento da hipertensão.

Além da redução da pressão arterial, duas participantes também relataram redução da prescrição medicamentosa: "não estou tomando o remédio". A orientação durante o programa foi para manter a prescrição medicamentosa, mas nestes casos, a redução da medicação foi realizada por orientação do médico que acompanhava as pacientes.

Outros benefícios relatados pelas participantes foram melhora da qualidade do sono com redução de medicação e diminuição da tensão muscular dos ombros e da região cervical.

\section{Dimensão Psicossocial}

Nesta dimensão são apresentadas questões pertinentes ao campo psicológico relacionadas à melhora do estado de humor, ao autoconhecimento e a autoestima e ainda reflexões voltadas ao âmbito social.

Quanto à melhora do estado de humor, foram apresentados vários relatos valorizando os benefícios que a atividade trouxe para o dia a dia das pessoas. Foram observadas afirmações relacionadas ao aumento de energia, melhora de disposição, mudança em aspectos da vida abordando um recomeço, maior disposição para se cuidar, diminuição de estresse, melhora de quadro depressivo, presença de felicidade e melhora de estado de ânimo de maneira geral: "O bumor da gente melhora", "O estresse também, parece que diminui, eu tive uma boa melhora, eu era bem nervosa, ai eu fiquei mais calma", "Agora eu estou feliz".

As afirmações acima vêm ao encontro com o que apontam os estudos que relacionam os efeitos da prática de Ioga em quadros depressivos ${ }^{6}$, em pessoas com ansiedade ${ }^{7}$ e na melhora dos estados de ânimo em geral ${ }^{23-24}$.

$\mathrm{O}$ processo de autoconhecimento se apresenta quando os participantes apontam para suas reflexões observando sua postura, sua alimentação, sua respiração, sua maneira de sentar, levantar e deitar, demonstradas nas seguintes expressões: "Começa a rever", "começa pensar tudo diferente", "começa querer mudar outras coisas" e "Isso faz você refletir outros pontos também". A prática de Ioga, em função de suas características, nos permite entrar em contato com o silêncio e isso pode propiciar momentos nos quais se torna possível um olhar interior, promovendo a auto-observação.

Os princípios éticos e morais da Ioga, primeiros passos do Yoga Sutra de Patãnjali ${ }^{25-26}$, são componentes naturalmente presentes no ambiente de aula 
que podem ser incorporados pelos praticantes o que possivelmente faz desencadear um processo de revisão de valores e reflexão possibilitando o autoconhecimento.

A estratégia utilizada de oferecer um café da manhã comunitário propiciou a exposição do grupo ao componente social. O destaque dado pelas participantes quanto à delicadeza, atenção e educação dos professores e monitores, a possibilidade de poder retribuir para quem te oferece algo e ter o reconhecimento dos benefícios recebidos são elementos ligados ao desenvolvimento do sentido de gratidão.

\section{CONSIDERACִÕES FINAIS}

Os resultados apresentados apoiam a hipótese inicial deste estudo, de que os conteúdos dos relatos poderiam expressar os benefícios que o programa proporcionou aos participantes, benefícios estes desde a perspectiva da saúde física, mental e social à mudança nos hábitos de vida, autoconhecimento e modo de se expressar e se relacionar. Num contexto mais amplo, as ideias que foram apresentadas refletem o contexto do grupo, mesmo quando foi expressa de maneira individualizada.

Os dados apontam para a existência de uma perspectiva em saúde que os sistemas de registros e avaliações médicas não conseguem enxergar, ou seja, benefícios que o modelo cartesiano de atenção à saúde não alcança mas que se mostram extremamente importantes a nível individual, conforme apontados nos relatos.

As práticas corporais como a Ioga baseiam-se na integralidade do sujeito, abordando-o em sua totalidade. Essa característica proporciona um suporte humanizado com foco na pessoa como um todo e não apenas na doença, associado a uma escuta acolhedora ${ }^{27}$. A Ioga conduz o praticante a uma reflexão sobre seu modo de vida, seus ideais e sobre si mesmo, levando-o ao autoconhecimento ${ }^{2}$ e criando um contexto extremamente favorável para mudanças nos hábitos de vida. Essas mudanças foram além da expectativa inicial do Programa Ioga e Hipertensão que era a de reduzir a pressão arterial.

Os relatos das mulheres sobre a experiência corporal poderiam estar descritas em seus prontuários clínicos, até porque esses efeitos não são proporcionados apenas pela prática da Ioga, mas decorrentes de todo um processo terapêutico que envolveu uma equipe multiprofissional com médicos, enfermeiros, auxiliares de enfermagem e educadores físicos, cujo tratamento envolveu a combinação do esquema medicamentoso com a intervenção terapêutica adjuvante, os quais, conjuntamente desencadearam nos resultados obtidos.

Em face das informações amealhadas, práticas corporais como a Ioga poderiam ser introduzidas nos serviços de atenção básica em saúde, por poderem ser realizadas em espaço físico restrito, apresentarem baixo risco de intercorrências, ter custo muito baixo para o serviço e contribuir fortemente para o sucesso terapêutico, podendo reduzir em $12 \%$ os custos anuais do município com a demanda pelos serviços de saúde, como observado com grupos que realizavam atividades físicas regulares ${ }^{28}$.

Para que a inserção destas práticas seja efetiva torna-se necessária também a qualificação de profissionais de Educação Física ou outros profissionais da área da saúde para que possam ofertar esses serviços na atenção básica. 
A respeito da limitação deste estudo, recorremos a Morgan (1997) para ressaltar que em dinâmicas de grupo focal alguns participantes podem adotar certa postura de conformidade, deixando de fornecer informações que possivelmente seriam expostas em entrevistas individuais. Embora os pesquisadores/moderadores tenham promovido um ambiente favorável à participação efetiva de todas as participantes, observamos que algumas mulheres expressaram suas opiniões com menor frequência em relação a outras. Tal limitação pode ser superada, em estudos futuros, com a inclusão da técnica complementar de entrevista, permitindo captar de modo mais individualizado pontos de vista de participantes que, porventura, não se sintam a vontade para expressarse coletivamente.

Em suma, os objetivos do estudo foram alcançados com a dinâmica do grupo focal, sendo possível observar inúmeros resultados a partir da perspectiva das participantes, que evidenciaram que o método de pesquisa qualitativa contribui para a compreensão dos benefícios da Ioga ao dar voz aos participantes. Com esses resultados os autores sugerem que as observações dos grupos focais sejam objeto de registro sistemático nos prontuários clínicos das participantes, bem como o treinamento e a capacitação de profissionais especializados para atuação no sistema público de saúde com a prática de Ioga.

\section{Agradecimentos}

Fundação para o Desenvolvimento de Bauru (FUNDEB) e Conselho Nacional de Pesquisa (CNPq) pelo apoio financeiro.

\section{REFERÊNCIAS}

1. Silva AM, Medeiros FE, Lazzarotti Filho A, Silva APS, Antunes PC, Leite JO. Corpo e Experiência: para pensar as práticas corporais. In: Práticas corporais no contexto contemporâneo: (in)tensas experiências. Org. Falcão JLC e Saraiva MC. Florianópolis: Copiart, 2009.

2. Feuerstein, G. A Tradição do Yoga. São Paulo: Pensamento, 1998.

3. Gnerre MLA. Identidades e paradoxos do yoga no Brasil: caminho espiritual, prática de Relaxamento ou atividade física? Fronteiras. 2010;12(21):247-70.

4. Mizuno J, Monteiro HL. An assessment of a sequence of yoga exercises to patients with arterial hypertension. J Bodyw Mov Ther. 2013;17: 35-41.

5. Malhota V, Singh S, Tandon OP, Sharma SB. The beneficial effect of yoga in diabetes. Nepal Med Coll J. 2005;7(2):145-7.

6. Woolery A, Myers H, Sternlieb B, Zeltzer L. A yoga intervention for young adults with elevated symptoms of depression. Altern Ther Health Med. 2004;10(2):60-3.

7. Michalsen A, Grossman P, Acil A, Langhorst J, Ludkte R, Esch T, et al. Rapid stress reduction and anxiolysis among distressed women as a consequence of a three-month intensive yoga program. Med Sci Monit. 2005;11(12):555-61.

8. Khalsa SB. Treatment of chronic insomnia with yoga: a preliminary study with sleepwake diaries. Appl Psychophysiol Biofeedback. 2004;29(4):269-78.

9. Ginzburg C. Sinais: raízes de um paradigma indiciário. In: __ . Mitos, emblemas, sinais: morfologia e história. São Paulo: Companhia das Letras. 1989: 143-179.

10. Gatti BA. Grupo focal na pesquisa em Ciências Sociais e Humanas. Brasília: Líber Livro, 2005.

11. Morgan DL. Focus groups as qualitative research. $2^{\text {a }}$ ed. Newbury Park, CA: Sage, 1997.

12. Patton MQ. Qualitative evaluation and research methods. $3^{\mathrm{a}}$ ed. Newbury Park, CA: SAGE, 2002.

13. Bardin L. Análise de conteúdo. Lisboa: Edições 70, 2000. 
14. Triviños ANS. Introdução à pesquisa em Ciências Sociais: a pesquisa qualitativa em educação. São Paulo: Atlas, 1987.

15. Castellano SM, Paulo TRS, Souza AGD, Santiago LB, Simões R. Ginástica funcional no programa quero mais saúde: motivos de adesão e permanência dos clientes atendidos numa cooperativa médica de Uberaba/mg. Coleção Pesquisa em Educação Física. 2012;11(5):55-62.

16. Iyengar BKS. A luz da Yoga. $9^{a}$ ed. São Paulo: Cultrix, 2007.

17. Cowen VS, Adams TB. Physical and perceptual benefits of yoga asana practice: results of a pilot study. J Bodyw Mov Ther. 2005;9:211-9.

18. Clay CC, Lloyd LK, Walker J L, Sharp KR, Pankey RB. The metabolic cost of hatha yoga. J Strength Cond Res. 2005;19(3):604-10.

19. Gonçalves LC, Vale RGS, Barata NJF, Varejão RV, Dantas EHM. Flexibility, functional autonomy and quality of life (QoL) in elderly yoga practitioners. Arch Geront Geriat. 2011;53(2):58-162.

20. Saper RB, Sherman KJ, Cullum-Dugan D, Davis RB, Phillips RS, Culpepper L. Yoga for chronic low back pain in a predominantly minority population: a pilot randomized controlled trial. Altern Ther Health Med. 2009;15(6):18-27.

21. Williams K, Abildso C, Steinberg L, Doyle E, Epstein B, Smith D, et al. Evaluation of the effectiveness and efficacy of Iyengar yoga therapy on chronic low back pain. Spine. 2009;34(19):2066-76.

22. Bussing A, Ostermann T, Ludtke R, Michalsenz A. Effects of Yoga Interventions on Pain and Pain-Associated Disability: A Meta-Analysis. J Pain. 2012;13(1);1-9.

23. Shapiro D, Cline K. Mood changes associated with Iyengar yoga practices: a pilot study. Int J Yoga Therap. 2004;14:35-44.

24. Shapiro D, Cook IA, Davydov DM, Ottaviani C, Leuchter AF, Abrams M. Yoga as a complementary treatment of depression: Effects of trait and moods on treatment outcome. J Evid Based Complementary Altern Med. 2007;4(4):493-502.

25. Taimni IK. A Ciência do Yoga. Brasília: Editora Teosófica, 1996.

26. Mehta R. Yoga: a arte da integração (Comentários sobre os yoga-sutras de Patañjali). Brasília: Teosófica, 1995.

27. Freitas FF, Brasil FK, Da Silva CL. Práticas corporais e saúde: novos olhares. Rev Bras Ciênc Esporte. 2006;27(3):169-83.

28. Codogno JS, Fernandes RA, Sarti FM, Freitas Junior IF, Monteiro HL. The burden of physical activity on type 2 diabetes public healthcare expenditures among adults: a retrospective study. BMC Public Health (Online). 2011;11:275.

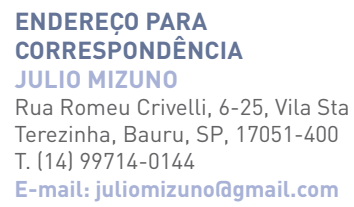

